\title{
SUR LES DEUX BORDS DE L'ATLANTIQUE SUD.
}

\author{
Th. Monod \\ Correspondant de 1'Institut \\ Professeur au Muséum National d'Histoire Naturelle \\ (Pêches Coloniales) \\ Directeur de 1'Institut Français d'Afrique Noire
}

On a insisté, et à juste titre, sur les caractères structuraux communs de l'Afrique et du Brésil, par exemple (Du Toit, 1927; Picard, 1939; de Martonne, 1946), et de fait, le schéma genéral est le même tant pour l'histoire stratigrafique (bouclier précambrien et couvertures sédimentaires épicontinentales ou non marines) que pour une morphologie relevant principalment de l'épirogenèse (bombements et cuvettes, developpement, au contact couverture-socle, des cuestas, etc.). Ces similitudes peuvent être dues à la séparation de masses primitivement contigües ou, plus probablement, dépuis surtout que sont connus les grand's traits du relief atlantique, aux oscillation verticales de surface structurellement homologues et à la disparition, relativement tardive (milieu du Tertiaire?) de l'Archihelenis de von Jhering (von Ihering, 1927, 1931; Gregory, 1929).

Les faunes et les flores terrestres et dulcaquicoles sont, dans l'ensemble, profondément différentes, et si les eaux douces d'Amérique du Sud et d'Afrique ont en commun les Dipneustes, les Ostéoglossidés, les Characidés, les Cichlidés, les Cyprinodontidés, les Nandidés, les Siluriformes, l'identité ne descend jamais au niveau du genre et à plus forte raison de l'éspèce et il n'y a pas de Preciliidés à l'Est, pas de Cypriniformes ni d'Anabantidés, ni de Mormyriformes, ni d'Ophiocephalidés à l'Ouest de l'Atlantique Sud.

Les deux continents sont depuis assez longtemps disjoints pour avoir connu, dans leur peuplement une histoire largement distincte. Et les voici séparés par un océan qui, en son point le plus étroit, a encore 2840 kilomètres de large et qui a si efficacement cloisonné l'oecóumène, qu'on a pu considérer l'Atlantique, franchi seulement à l'époque moderne (1) comme ayant constitué l'obstacle majeur opposé dans le passé à la diffusion des cultures et aux migrations humaines (Monod, 1944).

(1) Aux latitudes moyennes ou basses. 
Mais cet énorme océan qui est demeuré si longtemps infranchissable à l'homme, cette "Mer ténébreuse", sans archipels, sans voiles et sans "vis à vis", qui devait rester des millénaires durant l'effroi des marins, et sans doute des deux cotés, il n'est peut-être pas, biologiquement, aussi "imperméable", aussi "étanche" qu'on le pourrait imaginer, voir, par exemple: $1^{\circ}$, pour le Brésil: Rawitscher, 1944; Dansereau, 1947; 2\%, pour les Antilles et la Floride: Stehlé, 1935; Davis, 1940; Kurz, 1942; 3, pour 1'Afrique de l'Ouest: Muir, 1937; Trochain, 1940; Jaeger, 1949; Savory, 1949.

\section{BRESIL}

\section{AFRIQUE OCCIDENTALE}

\section{I. $P \mathrm{~L} A \mathrm{~A} E \mathrm{~S}$.}

Salicornia gaudichaudiana

Philoxerus vermiculatus

Sesuvium portucalastrum

Sporobolus virginicus

Stenotaphrum secundatum (=S. americanum)

Paspalum vaginatum

Conocaspus erectus

Hydrocotyle umbellatus

Tclanthera maritima

Canavalia obtusifolia

Ipomaea stolonifera $(=I$. littoralis $)$

Ipomaea pescaprae $(=I$. littoralis)

Remirea maritima

Scaevola Plumieri

Chrysobalanus icaco

Sophora occidentalis ( $=S$. tomentnea)

Olivancellaria spp. (auticularia, brasiliensis)

Donax hanleyanus

Emerita brasiliensis ( $=E$. emerita $\mathrm{H}$. Milne-Edw. nec Linné
Salicornia spp. (senegalensis, praecox)

Philoxerus vermiculatus

Sesuvium portucalastrum

Sporobolus virginicus et Sp. spicatus

Stenotaphrum secundatum

Paspalum vaginatum

Conocarpus erectus

Hydrocotyle asiatica

Telanthera maritima

Canavalia obtusifolia

ipomaea pes-caprae

Inomaea stolonifera

Remirea maritima

Scaevola Plumieri

Chrysobalanus orbicularis

Sophora occidentalis

Olivancellaria spp. (nana, hiatula)

Donax rugosus

Hippa cubensis

\section{II. $\mathrm{MAN} G \mathrm{R} O \mathrm{~V}$.}

Rhizophora racemosa

Avicennia nitida

Laguncularia racemosa

Fibiscus tiliaceus

Acrost:chum aureum
Rhizophora racemosa

Avicennia nitida

Laguncularia racemosa

Hibiscus tiliaceus

Acrostichum aureum

(1) Cf. N, Y. Acad. Sc. Scient. Surv. Porto-Rico, XV, 1935, p. 217 
Callinect $2 s$ sapidus

Collinectes marginatus

Panopeus Herbsti

Uca maracoani, leptodactyla, pugnax, etc...

Balanus amphitrite

Chtamalus stellatus

Gryphaea parasitica

Goniopsis cruentata

Cardisoma guanhumi

Casmerodius albus

Cicindela sp. (alba?)

Ocypode albicans
Callinectes spp.

Callinectes marginatis

Panopeus africanus

Balanus amphitrite

Chtamalus stellatus

Gryphaea gasar

Goniopsis cruentata

Cardisoma armatum

Casmerodius albus

Habrodera nitidula, Cicindela

senegalensis et clathrata.

Ocypode hippeus

\title{
III. L I T T O R A R O C H E $\mathrm{X}$.
}

\author{
Branchiostoma caribbeum (2) (*) \\ Mytilus perna \\ Thais haemastoma \\ Littorina lineata \\ Echinometra lucunter \\ Balanus tintinnabulum \\ Ligia exotica
}

Branchiostoma africae

Mytilus perna

Thais haemastoma

Littorina punctata

Echinometra lucunter

Balanus tintinnabulum

Ligia exotica

On pourrait multiplier les exemples. Ceux-ci suffisent à établir, et pour des groupes très variés d'organismes littoraux, une similitude de "tonalité" pouvant aller jusqu'à des identités spécifiques.

Ce qui, d'ailleurs, ne saurait faire oublier, par ailleurs les substantielles différences qui justifient la distinction de deux domaines faunistiques littoraux bien distincts: Amérique tropicale atlantique et Afrique occidentale présentant chacun une forte endémicité spécifique, et souvent générique.

La faune de l'Atlantique sud-américain au moins jusqu'à Rio, est un écho appauvri d'une faune chaude et riché celle des Antilles, héritière, avec l'Indonésie, encore qu'à un moindre degré, de la Tethys du début du Tertiaire, à caractère tropical très accusé (Coraux, etc.). Ajoutons que, pour certains (v. Ihering, 1931 p. 379), l'irruption vers le Nord des eaux de la Nereis australe ne se serait produite quau Quaternaire moyen, amenant Pingouins, Otaries et Baleines et faisant reculer la mangrove, avec son Ostraea parasitica, de 12 degrés, du Rio Negro à la frontière de l'Etat de Santa Catharina.

(2) M. Dansereau cite, naturellement, cet Amphioxus comme habitant des sables associés au littoral rocheux.

(*) Recentemente, SAWAYA, P. \& CARVALHO, J. de P. (1950, p. 235) reconheceram que a espécie de Branchiostoma do nosso litoral, anteriormente classificada como $B$. caribaeum, pertence à espécie $B$. platae, conforme BIGELOW \& FARFANTE (1948, p. 16) tinham indicado. N. da R. 

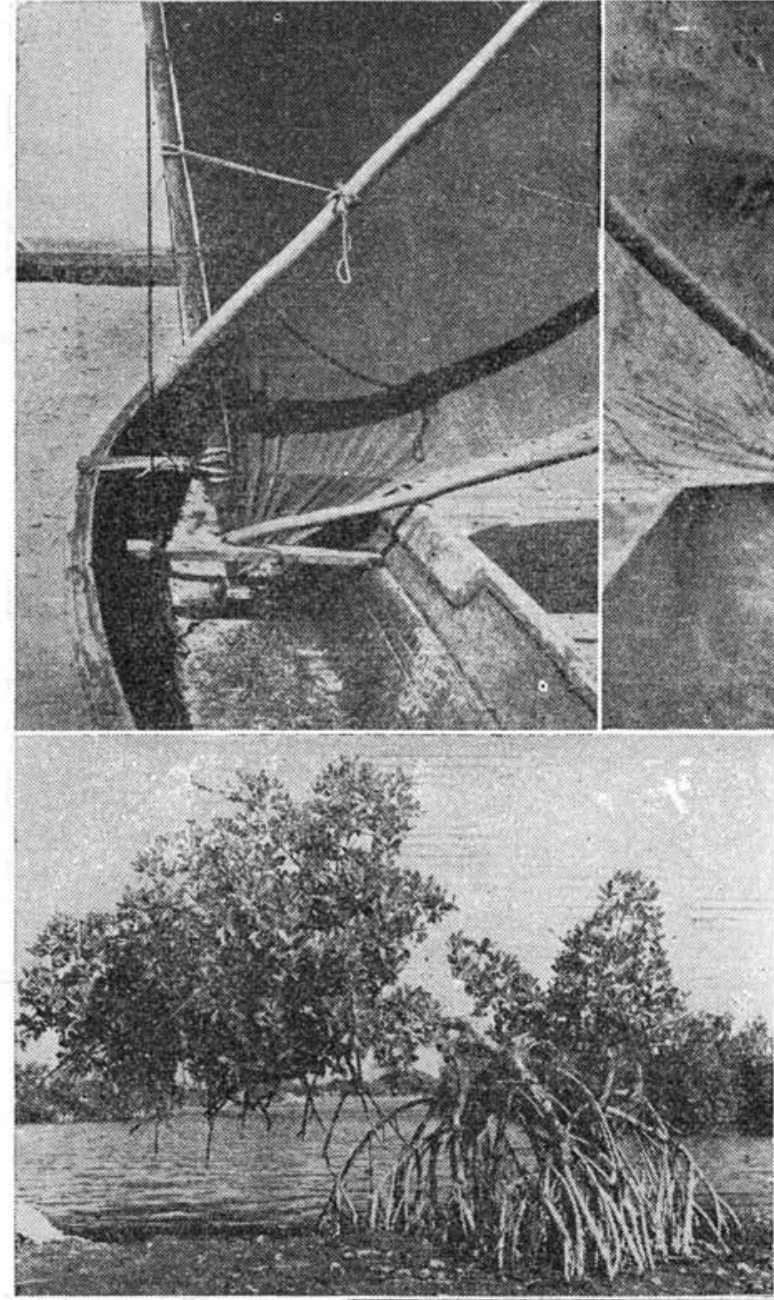

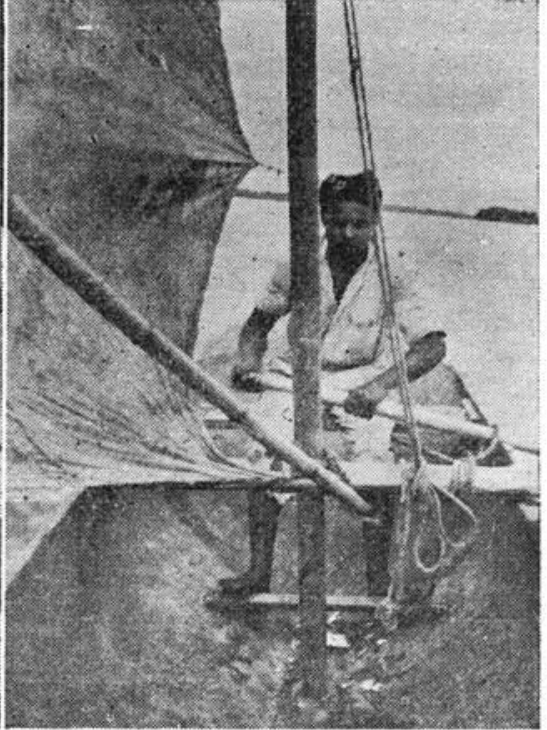

ALGUMAS NOTÁVEIS ANALOGIAS

1. Canòa da tríbu Léboue, do Senegal, Clichê I. F. A. N.; G. Labitte.

2 - Canòa de pesca de Ca. nanéia, Brasil (litoral sul do E. de S. Paulo). Clichê I. P. O.; W. Besnard.

3 - Rhizophora racemosa G. F. W. Meyer, perto de M'Bour, Senegal. Clichẻ I. F. A. N.; G. Labitte.

A-Mesmo tipo de vege. tação da laguna de $\mathrm{Ca}$ nanéia, Brasil (litoral sul do E. de S. Paulo). Clichẻ I. P. O.; W. Besnard.

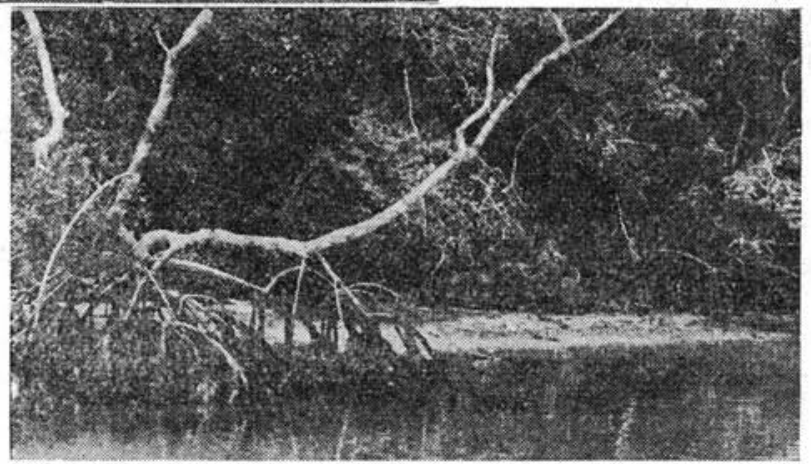


La faune de l'Afrique occidentale n'est plus en contact avec un domaine comparable aux Antilles puisque la Méditerranée a trés largement perdu ce caractère tropical, "indopacifique" qu'elle avait au début du Tertiaire; elie est cernée, par le Nord comme par le Sud, par deux faunes tempérées qui en rétrécissent le domaine et en influencent la composition.

On sait que Sven Ekman (1935) a insisté sur la pauvreté de la faune marine ouest-africaine, le très petit nombre des genres endémiques (1), et l'importance des caractères négatifs comme l'absence de récífs madréporiques véritables et de leur faune concomitante; le même auteur conclut que le domaine ouest-africain ne saurait constituer une division majeure équivalant à l'indopacifique ou à la tropico-américaine; c'est un terme intermédiaire relié aux autres par des communautés, génériques (Indo-pac., Médit., Amér.) ou spécifiques (Médit., Amér. à un moindre degré).

Si cette forte dyssymétrie trouve dans l'histoire géologique l'explication de sa genèse, la situation actuelle physiographique (géographie, nature des côtes, etc...) et surtout hydrologique, rend pleinement compte des faits contemporains dans leur apparente stabilité.

Un coup d'oeil même rapide jeté sur les cartes du "Meteor" ou sur l'excellente synthèse qu'en offre le manuel classique de Schott (1942), montre en effet combien, même à latitude égale, les deux bords du même océan sont différents.

Rappelons quelques exemples:

a) Précipitations: l'isohyète de $500 \mathrm{~mm}$. ne touche pas la côte américaine du Canada à Bahia Blanca, alors que plus de la moitié du littoral ouest-africain se trouve à l'intérieur de cette ligne, la $1 / 2$ recevant moins de $250 \mathrm{~mm}$., le $20^{\circ} \mathrm{W}$. entre $30^{\circ} \mathrm{N}$. et $30^{\circ} \mathrm{S}$. traverse, 2 larges aires sèches (250) séparés par un couloir pluvieux équatorial. L'Atlantique oriental est sec, l'occidental est humide.

b) Couleur de l'eau, etc.: eaux plus bleues à l'Ouest qu'à l'Est où le développement des eaux vertes, en particulier de 2 "langues" S.E.-N.W. (australe) et N.E.-S.W. (boréale), en rapport entre la circulation, semble l'être aussi avec la teneur en sels dissous, en plankton, en productivité.

c) Salinité: fortes salures à I'Ouest, un bon morceau de cśte brésilienne est à 36 et même $37 \mathrm{o} / \mathrm{oo}$; or cette dernière valeur ne touche nulle part la côte d'Afrique et les eaux à 36 elles mêmes, son limitées à la partie saharo-marocaine. Toute la partie équatoriale est à 35 et s'abaisse, au fond du golf de Guinée à 20 o 00 .

(1) Par exemple: Atlantotlos (Brachyure). Pusionella (Gastéropode). Rhopalodina (Holothurie). 
d) Températures de surface: la zone chaude à $25^{\circ}$ de moyenre annuelle, étroite à l'Est (Dakar à Congo) s'élargit considérablement vers l'Ouest (Floride à Caravellas). On comprend qu'il n'y ait guère place pour une véritable faune tropicale de type Antilles-Indonésie en Afrique occidentale, de plus qu'au facteur température s'ajoute une faible salinité, des substratums souvent peu favorables (sable et vase) etc...

e) Anomalies thermiques en surface: positives à l'Ouest, sur une grande surface (jusqu'à $+3^{\circ}$ ), négative a l'Est (jusqu'à $-5^{\circ}$ côte saharienne et $-9^{\circ}$ côte du désert du Namib).

f) Circulation: on connait le schéma et le transport général, par les deux flux Nord et Sud-équatorial, des eaux africaines vers 1'Amérique: la bouteille Schott $\mathrm{n}^{\circ} 6$ lâchée le 7 déc. 1880 par 29.24'S. et $33^{\circ}$ E. a été retrouvée le 11 août 1882 à Alcobaça, Brésil, par 17\%30'S, et $39^{\circ} 10^{\prime} \mathrm{W}$. ayant parcouru 4120 milles en 612 jours (moyenne: 6,7 milles par jour); la bouteille Gilchrist n² 296 lâchée le 11 juin 1900 à $261 / 2$ milles à l'Ouest de Cape Point (Afrique du Sud) était trouvée le 14 juillet 1901 sur la côte de Pernambuco, ayant fait plus de 2400 milles en 398 jours (moyenne: plus de $81 / 2$ milles par jour); enfin une bouteille lâchée dans la Baie de la Table le 29 avril 1909 se retrouvait le 10 février 1911 aux Bahamas (5.000 milles, moyenne: 9 milles par jour).

De profondes dyssymétries séparent les deux côtes. Est et $\mathrm{Ou}$ est, d'un même continent, celles aussi d'un même Océan. Il reste quand même que depuis le XV siècle, l'Atlantique est devenu in moyẹn de liaison extrèmement efficace entre l'Amérique du Sud et l'A. frique: Il n'est pas besoin de rappeler combien celle-ci est demeurée vivante à São Luiz do Maranhão, à Bahia, à Recife, jusqu'à PortoAlegre, et les étroites relations, même familiales, qui unissent encore le Dahomey et le Brésil.

D'autres causes doivent aujurd'hui resserrer les liens entre les pays bordiers d'un même océan. Et quel sujet pourrait plus efficacement les unir dans une amicale et féconde collaboration que les études ayant précisément pour objet l'océan même qui à la fois les sépare et les rapproche?

L'Atlantique des navires négriers a fait place à celui des bateaux océanographiques. Il est donc désormais légitime, je dirais plus, nécessaire, que les recherches marines, des deux côtés d'un même océan, se fassent de façon synchronique et parallèle.

Les outils de cette recherche, à tant d'égards si importante puisqu'elle conditionne tous les progrés futurs de l'exploitation rationelle des richesses marines, se forgent peu à peu. Au Brésil, voici que le jeune Instituto Paulista de Oceanografia, sous l'active et savante diréction de mon cher collègue et ami W. Besnard, se met résolument au travail; en Afrique occidentale française, l'Institut 
Français d'Afrique Noire possède une section d'Océanographie et de Biologie Marine, avec un laboratoire dans l'île de Gorée près de Dakar et un autre à Abidjan (Côte d'Ivoire) pour l'étude des lagunes; un Comité local d'Océanographie et d'étude des côtes functionne sous la diréction de la Marine Nationale et l'Inspéction générale de l'Elevage et des Industries animales s'intéresse activement aux pêches maritimes.

Il faut que les chercheurs "atlantiques", ceux du Brésil comme d'Afrique occidentale entretiennent de cordiales relations, échangent des informations (1), des idées, ou des matériaux d'étude, se communiquent leur travaux. Le jour peut venir où des formes plus directes encore de collaboration s'avèreront possibles, par exemple l'organisation en commun de croisières océanographiques ou des échanges: de personnel scientifique.

C'est pour tous ceux qui ont à coeur le développement des recherches dans l'Atlantique tropical une grande joie que de voir se développer si heureusement l'océanographie brésilienne: portant son effort sur une région encore très peu étudiée à cet égard, elle ne manquera pas d'obtenir, si les moyens matériels nécessaires lui sont accordés, les résultats les plus précieux tant pour les sciences en cause (océanographie physique et biologie marine) que pour une meilleure exploitation des richesses marines.

Un vieux chroniqueur du XVI ${ }^{\circ}$ siècle nous décrit les embarcations des Azenègues de la côte saharienne: "Os seus batees he çinco paos de figueyra de inferno secos, saber huum de braça e mea em longo e assi os dous em cada ylarga de dous palmos menos, e estes tres som atadas com linhas... e ficam detras todos tres ygoaes, e adiante sae o da metade mais, que he mais comprido..." (Cenival et Monod 1938, p. 118-119 et note 239).

Il n'est pas difficile de reconnaître dans cet étrange radeau une jangada brésilienne, ce qui pose un insoluble problème ethnographique peut-être, mais demeure pour moi le symbole de l'amicale collaboration scientifique et technique qui doit s'intensifier entre riverains d'un commun océan, "l'Atlantique des jangadas".

\section{R E S U M O}

Neste trabålho, trata o autor da fauna e da flora das orlas do Atlântico Sul em que se situam o Brasil e a África Ocidental. Sô-

(1) Si l'Afrique entreprend, par exemple, des recherches sur les courants au moyen de bouteilles flottantes, elle devra en aviser le Brésil, sur les côtes duquel il est probable que seront retrouvés plusieurs de ces objets. 
bre ambas, com justa razão, se tem procurado evidenciar, frequentemente, a semelhança existente quanto ao aspécto geológico.

Em relação à fauna e à flora terrestres e de água dôce, recorda - autor que essa semelhança não ultrapassa o limite de familias, nada de comum existindo, nos dois continentes, quanto a gêneros $e$ espécies. Por outro lado, porém, a fauna e a flora das duas margens do Atlântico são muito parecidas, havendo mesmo, nesse "habitat", numerosos gêneros e espécies em comum, conforme se depreende da relação constante à pag. 30 .

Apezar disso, existem divergências bastante acentuadas, capazes de permitir, como de fato acontece, que se considere as duas regiões como biogeográficamente distintas.

A fauna das costas brasileiras do Norte, até a latitude do Rio de Janeiro, é integrada pelos remanescentes de uma fauna tropical rica, proveniente do mar de "Tethis", do início do Terciário. Em altas latitudes, deu-se, no Quaternário médio, a invasão de águas austrais do "Nereis", que trouxeram consigo fórmas de águas frias. De maneira diverșa, a fauna da África ocidental perdeu o seu caráter tropical, tanto no Norte como no Sul, exibindo representantes faunisticos de zonas temperadas.

A fauna da costa ocidental africana é muito pobre, fato êsse que pode ser explicado à luz da história geológica da região e em face da situação fisiográfica e hidrológica atual.

Há numerosos exemplos da assimetria reinante nas duas margens do Atlântico, dentre os quais se podem citar os seguintes:

a) precipitações muito mais abundantes no Atlântico ocidental, úmido, em confronto com o oriental sêco;

b) côr mais azulada do Atlântico ocidental, fato relacionado com a salinidade, com a riqueza do plancton e com a produtividade das águas;

c) salinidade muito maior nas margens ocidentais;

d) a zona quente $\left(25^{\circ} \mathrm{C}\right.$. em mëdia $)$ é muito mais extensa nas costas ocidentais;

e) existência de numerosas anomalias térmicas superficiais no Atlântico ocidental;

f) diferenças de correntes maritimas nas duas margens do Atlântico.

Apezar das profundas divergências constatadas* em ambas as margens, lembra o autor que desde o século XV, o Atlântico tornou-se um meio eficiente para se promover relações entre o Brasil e a África do Sul. Afirma, assim, que novas ligações devem unir, presentemente, os paises limitrofes de um mesmo oceano. Os navios negreiros de outrora devem ser substituidos por barcos oceanográficos, 
visando o estabelecimento de relações amigáveis e de uma fecunda colaboração.

Diz ainda o autor que, para serem eficientes, as pesquisas oceanográficas levadas a cabo nas duas orlas de um mesmo oceano, devem ser realizadas sincrônica e paralelamente. Os meios tendentes a alcançar tal objetivo já se esboçam desde que - diz o autor "no Brasil inicia-se tal trabalho através do Instituto Paulista de Oceanografia, e, na África Ocidental funciona o Institut Français d'Afrique Noire que já possue uma secção de Oceanografia e de biologia marinha, com um laboratório instalado na ilha de Go:ée perto de Dakar e outro nas cercanias de Abidjan (costa do Marfim) ;ara estudo das lagunas".

Julga, portanto, o autor que se torna imperiosa a manutençâo de relações cordiais e de intercambio entre os pesquisadores dos dois lados do Atlântico, afim de que chegue o dia em que se realizem cruzeiros oceanográficos em comum, com trocas de cientistas.

Pensa o autor ser extremamente interessante desenvolver a cceanografia no Brasil, pois a sua região atlântica é uma das menos conhecidas, podendo-se, através da pesquisa, obter resultados importantíssimos, tan to sob o ponto de vista da ciência pura como no que respeita a exploração racional das riquezas marinhas.

Recorda ainda o autor que existe uma descrição das embarcações dos Azenegues, da costa do Sahara, de autoria de um cronista. do século XVI que lembra muito as jangadas brasileiras. Provavelmente. existe ai um problema etnográfico, de palpitante interesse, mas que, na sua opinião, representa antes um simbolo da colaboração técnico-cientifica que, segundo o seu parecer, deve ser mantida entre as duas margens do Atlântico.

M. V.

\section{BIBLIOGRAFIA}

BIGELOW, H. B. E FARFANTE, I. P., 1948 - Lancelets, in Fishes of the Western North Atlantic. Mem. Sears Found. Mar. Res. n. ${ }^{\circ}$ 1, XVII, 576p Yale Univ. N. Haven, Conn.

CENIVAL, P. DE \& MONOD, T., 1938 - Description de la côte d'Afrique de Ceuta au Sénégal par Valentim Fernandez (1506-1507). Paris.

DANSEREAU. P., 1947 - Zonation et succession sur la Restinga de Rio de Janeiro. I. Halosère. Rev. Canad., Biol., VI, n. 3.

DAVIS, J. M. Jr., 1940 - The Ecology and Geologic Rôle of Mangroves in Florida. Pap. Tort. Lab., XXXII.

DU TOIT, A. L., 1927 - A geological Comparison of S. America with S. Africa. Carnegie Inst. Publ. 381.

EKMAN S., 1935 - Tiergeographie des Meeres. Le'pzig. 
GREGORY, J. W., 1929 - The Geological History of the Atlantic Ocean. Quart. Journ. Geol. Soc., LXXXV.

VON IHERING, H., 1927 - Die Geschichte des Atlantischen Ozeans, Jena.

VON IHERING, H., 1931 - Land bridges across the Atlantic and Pacific Oceans during the Kainozoic Era. Quart. Journ. Geol. Soc. LXXXVII, pt. 3.

JAEGER, P., 1949 - Le végétation. in: La presqu'île du Cap Vert. Institut Français d'Afrique Noire. Dakar.

KURZ, M., 1942 - Florida dunes and scrub, vegetation and geology. Florida Geol. Survey, Bull. n. ${ }^{\circ}$ 23, Tallahassee.

MARTONNE, E. DE. 1946 - Geographie Zonale: La zone tropicale. Ann. Géogr. LV, n. 297.

MONOD, T., 1944 - Au bord de l'Océan ténébreux: Atlantique et Afrique. 15 p. 23 figs. Saint Louis du Sénégal.

MUIR, J., 1937 - The Seed-drift of South Africa and some influences of Ocean Currents on the strand Vegetation. Bot. Surv. Mem. n. ${ }^{\circ} 16$. Pretoria.

PICARD, L., 1939 - Outline of the Tectonics of the Earth. Bull. Geol. Dept. Hebrew University, II, n. ${ }^{\circ}$ 3-4. Jerusalem.

RAWITSCHER, F. K., 1944 - Algumas noçōes sôbre a vegetação do litoral brasileiro. Bol. Ass. Geogr. Bras. n. 5.

SAVORY, M. J., 1949 - The vegetation of the Sea-shore, Salt-marshes and Sandbanks in the Lagos area. Com. Conf. Inst. Africanistes de l'Ouest Ibadan.

SAWAYA, P. E J. de P. CARVALHO, 1950 - On the Branchiostoma (Amphioxus) of the Coast of São Paulo. Bol. Fac. Fil. Ciênc. Letras. Zoologia n. ${ }^{\circ}$ 15. p. 235-237. São Paulo.

SCHOTT, G., 1942 - Geographie des Atlantischen Ozeans, 3e ed.

STEHLÉ, H., 1935 - Flore de la Guadeloupe et Dépendances - I - Essai d'Écologie et de Géographie botanique. Basse-Terre.

TROCHAIN, J., 1940 - Contr:bution à l'étude de la végétation du Sénégal. Mem. Inst. Français d'Afrique Noire. $11^{\circ} 2$. 